\section{$\Delta$ rkivoc

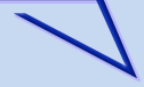

Archive for

Organic Chemistry
The Free Internet Journal

for Organic Chemistry
Paper

Arkivoc 2017, part iii, 36-44

\title{
The synthesis of fluorine-containing endothiopeptide analogs by the reaction of perfluorinated dithiocarboxylic acid amides with esters of $\alpha$-amino acids and dipeptides
}

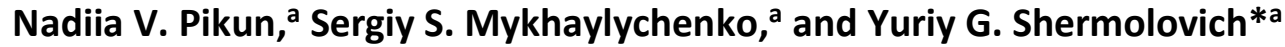

anstitute of Organic Chemistry, National Academy of Sciences of Ukraine, 5, Murmanska, 02094, Kiev, Ukraine Email: sherm@ioch.kiev.ua

Received 08-18-2017

Accepted 10-27-2017

Published on line 11-29-2017

\section{Abstract}

One of the main approaches to the change and increase of biological activity of pharmacologically important peptides is the incorporation of non-natural amino acids into their structure. ${ }^{1} \mathrm{~A}$ new method for the synthesis of fluorinated endothiopeptide analogs, containing the thiocarbonyl groups and fluorine atoms in the main peptide chain, is described. The new method consists of thioacylation reactions of the esters of corresponding $\alpha$-amino acids and dipeptides with amides of perfluorinated dithiocarboxylic acids.

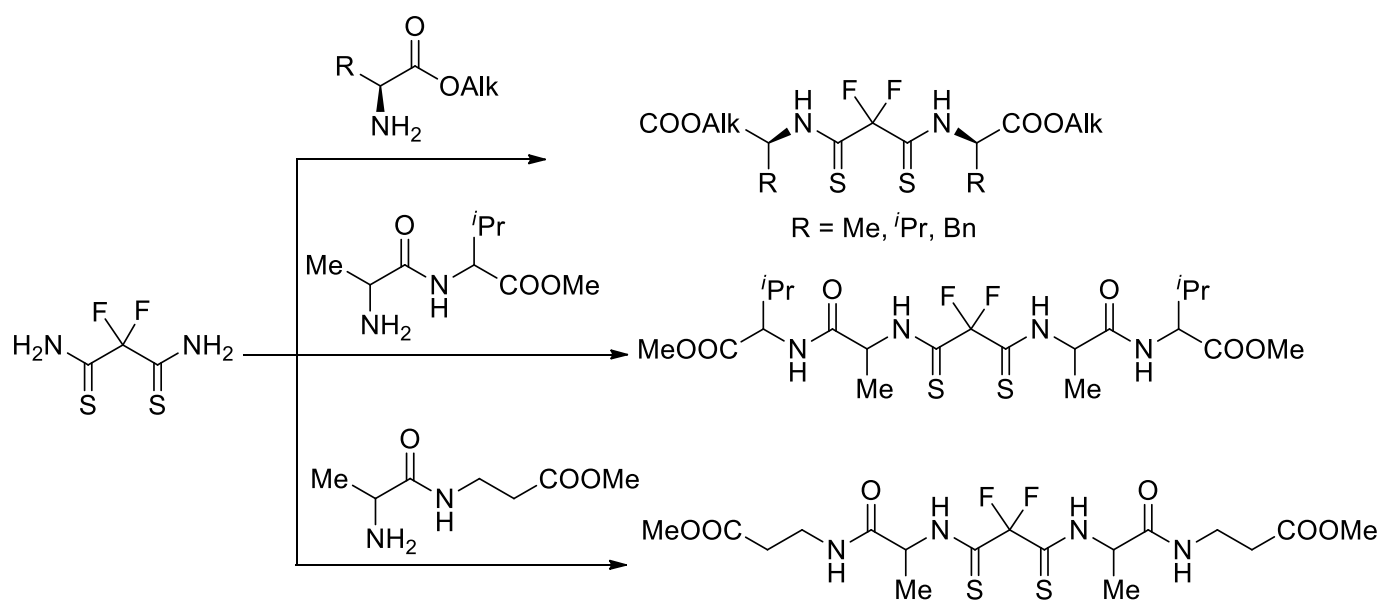

Keywords: Fluorinated thioamide, thioacylation, amino acid, endothiopeptide 


\section{Introduction}

One of the main approaches to changing and increasing the biological activity of pharmacologically-important peptides is the incorporation of non-natural amino acids into their structures. ${ }^{1}$ Peptides containing fluorinated amino acid residues ${ }^{2-4}$ and endothiopeptides, in which one of the peptidic bonds is replaced by a thiopeptidic bond, ${ }^{5-6}$ have been widely studied over prior decades. Thiopeptides have been found to possess a wide range of biological properties, including antibacterial ${ }^{7}$, anticancer ${ }^{8-12}$, antiplasmodial ${ }^{13-15}$, immunosuppressive ${ }^{16}$, renin-inhibitory ${ }^{17}$, RNA-polymerase-inhibitory ${ }^{18}$ and antifungal ${ }^{19}$ activities. In the design of pharmaceuticals, the introduction of a fluorine atom is used as part of strategy to increase the metabolic stability and lipophilicity of the peptide, which improved absorption and transport of the agent to its biological target. ${ }^{20-24}$

It is known that the synthesis of fluorinated peptide analogs is generally based on the use of amino acids containing fluorinated substituents on the side chain. Endothiopeptides are prepared by the thionation reaction of the amide's carbonyl group of the corresponding peptides..$^{25}$

The aim of our work was to research methods for the synthesis of new types of fluorinated thiopeptides containing the fluorine atoms in the main peptide chain. It is known that the fluorinated substituents $\left(\mathrm{CF}_{1} \mathrm{CF}_{2}\right)$ have been used as an isosteric analog of the amide functionality. ${ }^{26-27}$

\section{Results and Discussion}

Previously, we have shown that, in contrast to non-fluorinated analogs, amides of polyfluoroalkanethiocarboxylic acids can be mild thioacylating reagents for compounds containing a primary amino group, including alkyl amines and $\alpha$-amino acid esters. New fluorinated thiopeptide analogs (2) were obtained by the reaction of polyfluoroalkanethioamides $(\mathbf{1} \mathbf{a}, \mathbf{b})$ with $\alpha$-amino acid esters. ${ }^{28}$ In continuation of this work, we have found that 2,2,3,3-tetrafluoropropanethioamide (1) reacted with methyl $D L-\alpha$-alanyl-DLnorleucinate by refluxing in chloroform for $48 \mathrm{~h}$, affording compound (3) that was isolated in $65 \%$ yield after column chromatography (Scheme 1).

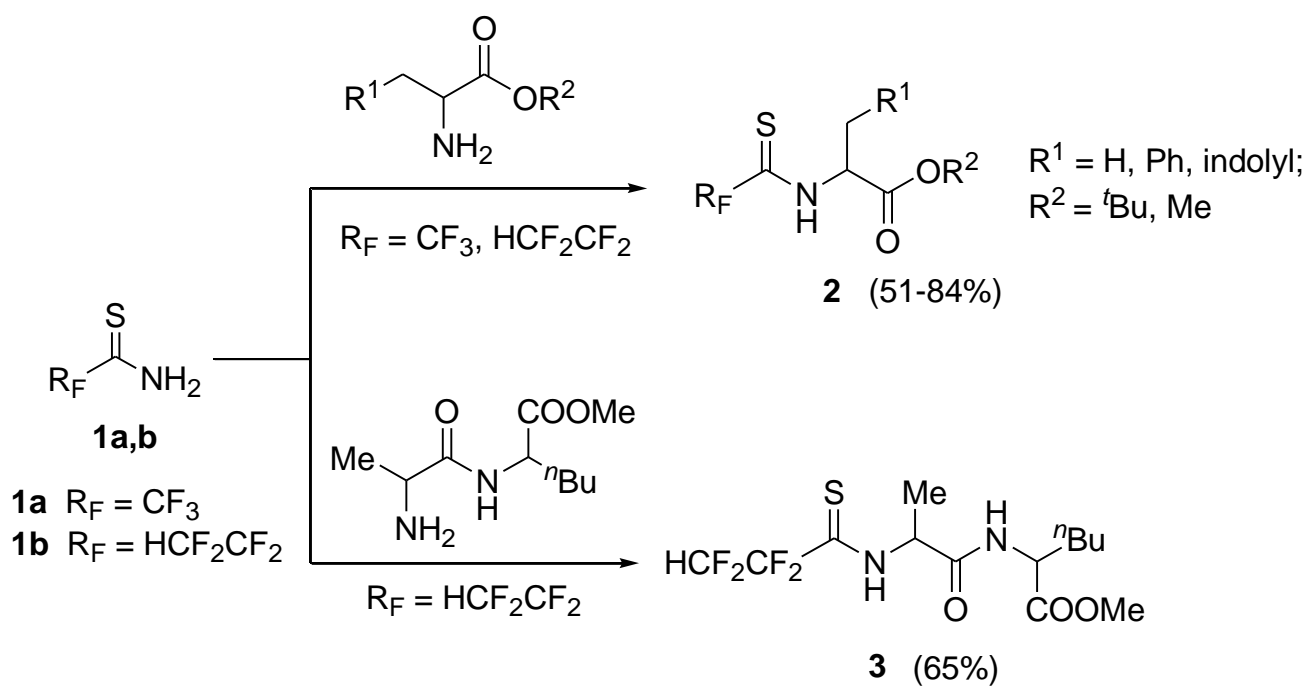

Scheme 1. Reactions of polyfluoroalkanethioamides (1a,b) with $\alpha$-amino acid and dipeptide esters. 
The main intent of this work, however, was the investigation of the preparation of polyfluorinated thiopeptide analogs containing a thioamide linkage in the middle of the chain. The thioamides of polyfluorinated dicarboxylic acids can be used as starting compounds for the preparation of such thiopeptide analogs. With this objective in mind, we have studied the possibilities to obtain thioamides by the thionation of amides of difluoromalonic and tetrafluorosuccinic acids.

Recently we have found that the thionation of 2,2,3,3-tetrafluorosuccinamide with phosphorus pentasulfide $\left(\mathrm{P}_{4} \mathrm{~S}_{10}\right)$, in the presence of hexamethyldisiloxane (HMDSO), afforded the corresponding 3,3,4,4tetrafluoropyrrolidine-2,5-dithione (4), probably as a result of the cyclization of 2,2,3,3tetrafluorosuccinthioamide. ${ }^{28}$ Conversely, the thionation of 2,2-difluoromalonoamide (5) under similar conditions afforded 2,2-difluoromalonothioamide (6) (Scheme 2).

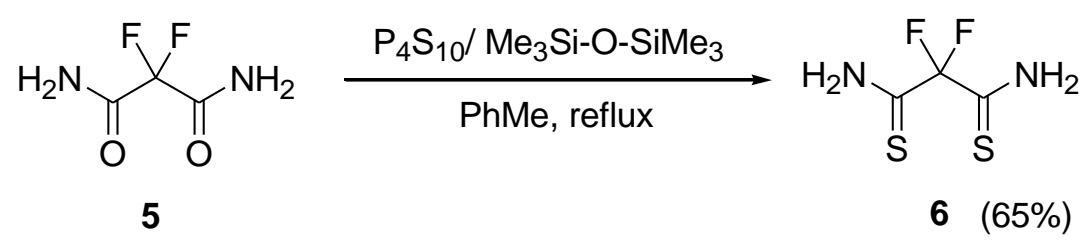

Scheme 2. Thionation of 2,2-difluoromalonoamide (5).

We have shown that 3,3,4,4-tetrafluoropyrrolidine-2,5-dithione (4) reacted with methyl L-phenylalaninate, methyl L-alaninate and isopropyl L-valinate at room temperature for 7 days, affording compounds (7a-c), which were isolated in 60-71\% yields after column chromatography (Scheme 3).

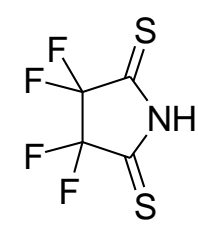

4

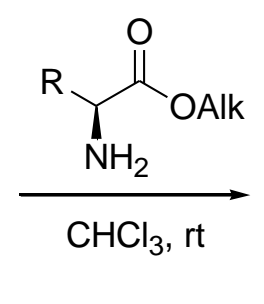

(1)<smiles>[R]C(NC(=S)C(F)(F)C(F)(F)C(=S)N[C@@H]([R])C(=O)O[AlH2])C(=O)O[AlH2]</smiles>

7a-c

$$
\begin{aligned}
& \mathrm{R}=\mathrm{Bn}, \text { Alk }=\mathrm{Me} \text { 7a }(71 \%) \\
& \mathrm{R}=\mathrm{Me}, \mathrm{Alk}=\mathrm{Me} \quad 7 \mathrm{~b}(60 \%) \\
& \mathrm{R}={ }^{i} \mathrm{Pr}, \mathrm{Alk}={ }^{i} \operatorname{Pr} \quad \text { 7c }(65 \%)
\end{aligned}
$$

Scheme 3. Reactions of 3,3,4,4-tetrafluoropyrrolidine-2,5-dithione (4) with $\alpha$-amino acid esters.

The reaction of 2,2-difluoromalonothioamide (6) with esters of $\alpha$-amino acids ( $L$-alanine, $L$-phenylalanine, L-valine) proceeds more slowly (20 days at room temperature) than in the case of 3,3,4,4tetrafluoropyrrolidine-2,5-dithione (4) giving compounds (8a-c) (Scheme 4). 


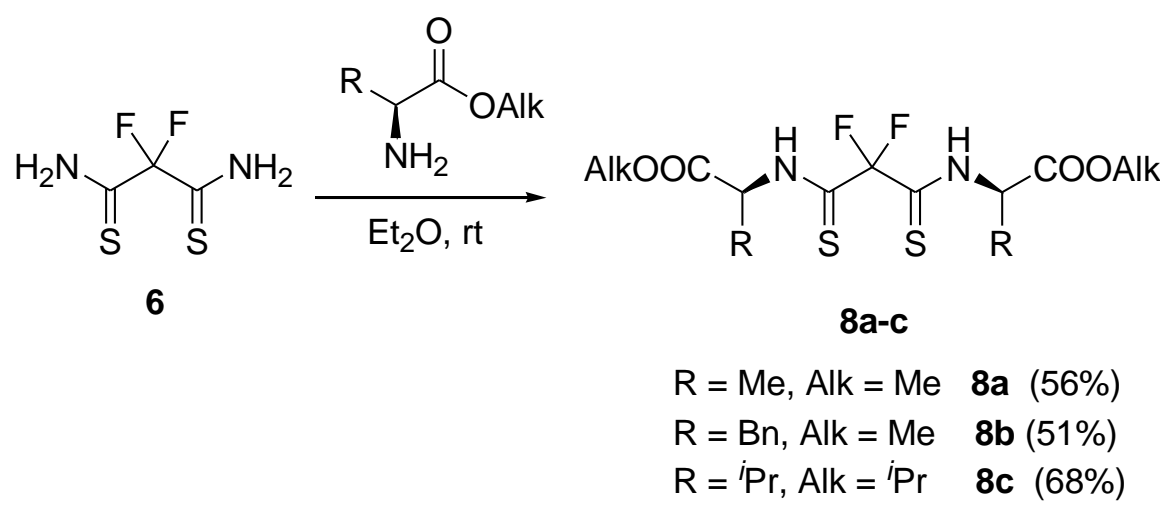

Scheme 4. Reactions of 2,2-difluoromalonothioamide (6) with $\alpha$-amino acid esters.

We have found that 2,2-difluoromalonothioamide (6) reacted with the methyl esters of dipeptides ( $D L-\alpha$ alanyl-DL-valinate, $D L-\alpha$-alanyl- $\beta$-alaninate) under more drastic conditions than with esters of $\alpha$-amino acids (i.e., heating in toluene at $80{ }^{\circ} \mathrm{C}$ for $50 \mathrm{~h}$ ) to afford compounds $(\mathbf{9 a , b})$, which were isolated after column chromatography as a mixture of diastereomers (Scheme 5). The ratio is 50:50 according to ${ }^{19} \mathrm{~F} \mathrm{NMR}$.

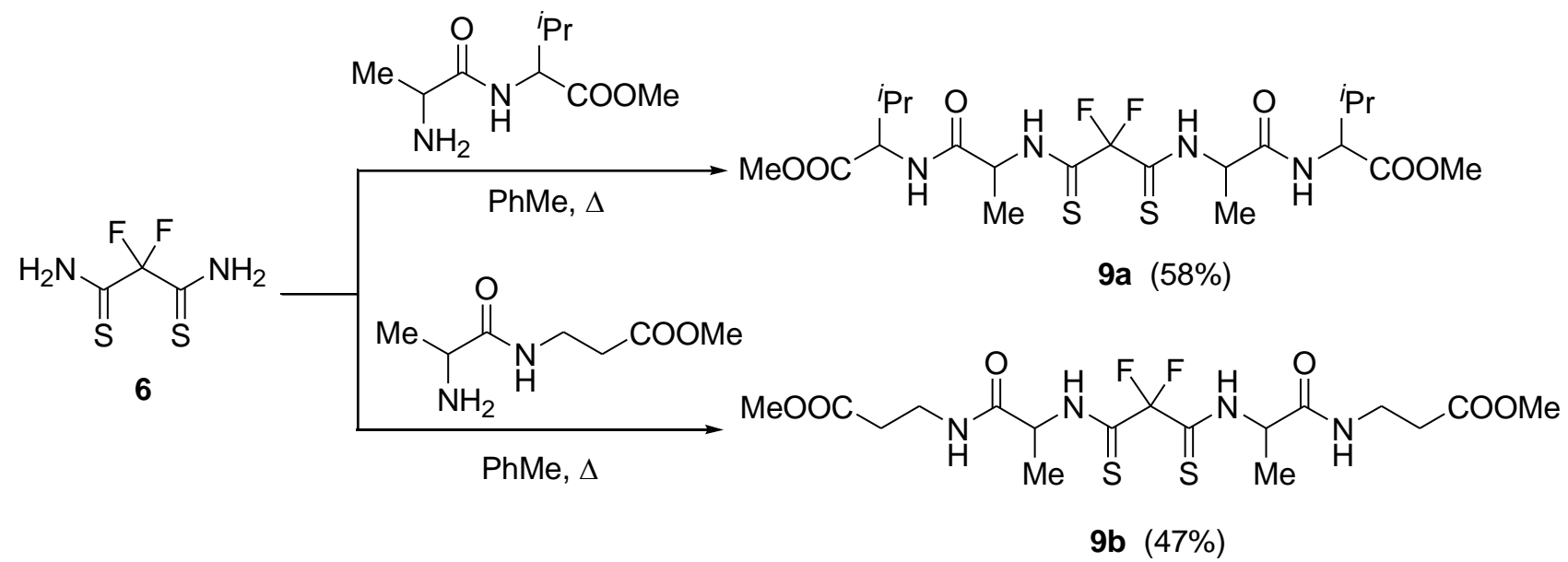

Scheme 5. Reactions of 2,2-difluoromalonothioamide (6) with dipeptide esters.

\section{Conclusions}

We have proposed a new method for the synthesis of fluorinated endothiopeptide analogs containing the thiocarbonyl group and fluorine atoms in the main peptide chain, by the thioacylation reaction of corresponding $\alpha$-amino acids and dipeptide esters with thioamides of perfluorinated dicarboxylic acids. As shown in the literature, introduction of a fluorine atom can be used as part of the strategy to increase the metabolic stability and lipophilicity of the peptide, which improves the absorption and transport of the agent to its biological target, thereby increasing the biological activity of pharmacologically-important peptides. 


\section{Experimental Section}

General. ${ }^{1} \mathrm{H}$ NMR and ${ }^{19} \mathrm{~F}$ spectra were recorded on a Bruker Avance 400 instrument at $399.98 \mathrm{MHz}$ and 376.47 MHz, respectively. ${ }^{13} \mathrm{C}$ NMR spectra were registered on a Bruker Avance 500 instrument at 125.75 $\mathrm{MHz}$. Tetramethylsilane $\left({ }^{1} \mathrm{H}\right.$ NMR: $\left.\delta 0.00 \mathrm{ppm}\right), \mathrm{CDCl}_{3}\left({ }^{13} \mathrm{C} \mathrm{NMR}: \delta 77.16 \mathrm{ppm}\right),\left(\mathrm{CD}_{3}\right)_{2} \mathrm{SO}\left({ }^{13} \mathrm{C}\right.$ NMR: $\delta 39.52$ ppm), $\mathrm{C}_{6} \mathrm{~F}_{6}\left({ }^{19} \mathrm{~F}\right.$ NMR: $\left.\delta-162.9 \mathrm{ppm}\right)$ were used as internal standards for ${ }^{1} \mathrm{H},{ }^{13} \mathrm{C}$ and ${ }^{19} \mathrm{~F} N M R$ spectra, respectively. LC-MS data were obtained using an ADSI MS, Agilent 1100 \DAD\MSD VLG1965 instrument (ESI, $70 \mathrm{eV})$. Optical rotation was measured on an automatic Anton Paar MCP 300 polarimeter. The elemental analyses were carried out at the Analytical Laboratory of the Institute of Organic Chemistry, National Academy of Sciences of Ukraine. Melting points were measured on a Boëtius heating block. Silica gel Merck 60 (70-230 $\mu \mathrm{m}$ ) was used for column chromatography. TLC was performed on Macherey-Nagel Polygram ${ }^{\circledR}$ Sil G/UV254 plates and visualized by exposure to UV-light or iodine vapor. The reaction progress was monitored by ${ }^{19} \mathrm{~F}$ NMR spectroscopy. All solvents were dried and distilled by standard procedures prior to use.

Methyl (2,2,3,3-tetrafluoropropanethioyl)-DL- $\alpha$-alanyl-DL-norleucinate (3). Methyl $D L-\alpha$-alanyl-DLnorleucinate $(200 \mathrm{mg}, 0.9 \mathrm{mmol})$ was added to a solution of 2,2,3,3-tetrafluoropropanethioamide (1b) (115 $\mathrm{mg}, 0.7 \mathrm{mmol}$ ) in chloroform $(15 \mathrm{~mL})$. The reaction mixture was refluxed for $48 \mathrm{~h}$. The solvent was then evaporated in vacuo and the crude product was purified by column chromatography on silica gel (hexane/EtOAc 7:3), to afford compound (3). Yield: 65\%. Yellow oil. $R_{\mathrm{f}}=0.33$ (hexane/EtOAc 7:3). ${ }^{1} \mathrm{H} N M R$ $\left(\mathrm{CDCl}_{3}, \delta \mathrm{ppm}\right): 0.90\left(\mathrm{~m}, 3 \mathrm{H},\left(\mathrm{CH}_{2}\right)_{2} \underline{\mathrm{H}}_{3}\right), 1.32\left(\mathrm{~m}, 4 \mathrm{H},\left(\mathrm{C}_{2}\right)_{2} \mathrm{CH}_{3}\right), 1.57\left(\mathrm{~d}, 3^{3} \mathrm{H}_{, \mathrm{H}} 7.2 \mathrm{~Hz}, 3 \mathrm{H}, \mathrm{CHC} \underline{H}_{3}\right), 1.70(\mathrm{~m}, 1 \mathrm{H}$, $\left.\underline{\mathrm{C}}_{\mathrm{A}} \mathrm{H}_{\mathrm{B}}\left(\mathrm{CH}_{2}\right)_{2} \mathrm{CH}_{3}\right), 1.88\left(\mathrm{~m}, 1 \mathrm{H}, \mathrm{CH}_{\mathrm{A}} \underline{H}_{\mathrm{B}}\left(\mathrm{CH}_{2}\right)_{2} \mathrm{CH}_{3}\right), 3.78\left(\mathrm{~s}, 3 \mathrm{H}, \mathrm{OCH}_{3}\right), 4.63(\mathrm{~m}, 1 \mathrm{H}, \mathrm{CH}), 4.96(\mathrm{~m}, 1 \mathrm{H}, \mathrm{CH}), 6.43(\mathrm{tt}$, $\left.{ }^{2} J_{\mathrm{H}, \mathrm{F}} 52.8 \mathrm{~Hz},{ }^{3} J_{\mathrm{H}, \mathrm{F}} 5.8 \mathrm{~Hz}, 1 \mathrm{H}, \mathrm{HCF}_{2}\right), 9.00$ (bs, $\left.1 \mathrm{H}, \mathrm{NH}\right) .{ }^{19} \mathrm{~F} \mathrm{NMR}\left(\mathrm{CDCl}_{3}, \delta \mathrm{ppm}\right):-120.3\left(\mathrm{dm},{ }^{2} \mathrm{~J}_{\mathrm{F}, \mathrm{F}} 252.6 \mathrm{~Hz}, 1 \mathrm{~F}\right.$, $\left.\mathrm{CF}_{\mathrm{A}} \mathrm{F}_{\mathrm{B}}\right),-121.5\left(\mathrm{dm},{ }^{2} \mathrm{~J}_{\mathrm{F}, \mathrm{F}} 252.6 \mathrm{~Hz}, 1 \mathrm{~F}, \mathrm{CF}_{\mathrm{A}} \underline{\mathrm{F}}_{\mathrm{B}}\right),-140.0\left(\mathrm{dm},{ }^{2} \mathrm{~J}_{\mathrm{F}, \mathrm{H}} 52.8 \mathrm{~Hz}, 2 \mathrm{~F}, \mathrm{HCF}_{2}\right) .{ }^{13} \mathrm{C} \mathrm{NMR}\left(\mathrm{CDCl}_{3}, \delta \mathrm{ppm}\right): 13.9$ $\left(\mathrm{s},\left(\mathrm{CH}_{2}\right)_{2} \underline{\mathrm{CH}}_{3}\right), 17.2\left(\mathrm{~s}, \mathrm{CH}-\mathrm{CH}_{3}\right), 22.3\left(\mathrm{~s}, \mathrm{CH}_{2}\right), 27.3\left(\mathrm{~s}, \mathrm{CH}_{2}\right), 32.2\left(\mathrm{~s}, \mathrm{CH}_{2}\right), 52.5\left(\mathrm{~s}, \mathrm{OCH}_{3}\right), 52.8(\mathrm{~s}, \mathrm{CH}), 53.9(\mathrm{~s}$, $\mathrm{CH}), 109.4-112.2\left(\mathrm{~m}, \mathrm{HCF}_{2} \mathrm{CF}_{2}\right), 170.1(\mathrm{~s}, \mathrm{C}=\mathrm{O}), 173.0(\mathrm{~s}, \mathrm{C}=\mathrm{O}), 185.3$ (t, $\left.{ }^{3} \mathrm{~J}_{\mathrm{C}, \mathrm{F}} 23.7 \mathrm{~Hz}, \mathrm{C}=\mathrm{S}\right) . \mathrm{MS}, \mathrm{m} / \mathrm{z}: 359$ [M-H] $]^{-}$. Anal. calcd for $\mathrm{C}_{13} \mathrm{H}_{20} \mathrm{~F}_{4} \mathrm{~N}_{2} \mathrm{O}_{3} \mathrm{~S}: \mathrm{C}, 43.33 ; \mathrm{H}, 5.59 ; \mathrm{N}, 7.77 ; \mathrm{S}, 8.90$. Found: $\mathrm{C}, 43.42 ; \mathrm{H}, 5.57 ; \mathrm{N}, 7.81 ; \mathrm{S}$, 8.97.

General procedure for the reaction of 3,3,4,4-tetrafluoropyrrolidine-2,5-dithione (4) with $\alpha$-amino acid esters. The respective amino acid ester $(2.3 \mathrm{mmol})$ was added to a solution of 3,3,4,4-tetrafluoropyrrolidine2,5-dithione (4) $(200 \mathrm{mg}, 1.0 \mathrm{mmol})$ in chloroform $(10 \mathrm{~mL})$. The reaction mixture was stirred at room temperature for 7 days. The solvent was then evaporated in vacuo and the crude product purified by column chromatography on silica gel, eluting with a mixture of hexane and ethyl acetate (4:1) to afford the corresponding polyfluoroalkanedithioyl derivative (7a-c). Compound (7a) was described by us early. ${ }^{28}$

Dimethyl $N, N^{\prime}-(2,2,3,3-t e t r a f l u o r o b u t a n e d i t h i o y l) ~ b i s(L-a l a n i n a t e)(7 b)$. Yield: $60 \%$. Yellow oil. $[\alpha]_{D}{ }^{20}-88.9^{\circ}$ (c=1, MeOH). $R_{\mathrm{f}}=0.39$ (hexane/EtOAc 4:1). ${ }^{1} \mathrm{H} \mathrm{NMR}\left(\mathrm{CDCl}_{3}, \delta \mathrm{ppm}\right): 1.51\left(\mathrm{~d}, 3^{3} \mathrm{H}_{\mathrm{H}} \mathrm{H} 7.3 \mathrm{~Hz}, 6 \mathrm{H}, 2 \times \mathrm{CH}_{3}\right), 3.81(\mathrm{~s}$, $\left.6 \mathrm{H}, 2 \times \mathrm{OCH}_{3}\right), 5.00(\mathrm{~m}, 2 \mathrm{H}, 2 \times \mathrm{CH}), 8.54$ (bs, $\left.2 \mathrm{H}, 2 \times \mathrm{NH}\right) .{ }^{19} \mathrm{~F} \mathrm{NMR}\left(\mathrm{CDCl}_{3}, \delta \mathrm{ppm}\right):-109.6\left(\mathrm{dm},{ }^{2} \mathrm{~J}_{\mathrm{F}, \mathrm{F}} 259.0 \mathrm{~Hz}\right.$, $\left.2 \mathrm{~F}, 2 \times \mathrm{CF}_{\mathrm{A}} \mathrm{CF}_{\mathrm{B}}\right),-110.5\left(\mathrm{dm},{ }^{2} \mathrm{~J}_{\mathrm{F}, \mathrm{F}} 259.0 \mathrm{~Hz}, 2 \mathrm{~F}, 2 \times \mathrm{CF}_{\mathrm{A}} \mathrm{CF}_{\mathrm{B}}\right) .{ }^{13} \mathrm{C} \mathrm{NMR}\left(\mathrm{CDCl}_{3}, \delta \mathrm{ppm}\right): 16.1\left(\mathrm{~s}, 2 \times \mathrm{CH}_{3}\right), 53.0(\mathrm{~s}, 2$ $\times \mathrm{CH}), 53.7\left(\mathrm{~s}, 2 \times \mathrm{OCH}_{3}\right), 109.0-114.2\left(\mathrm{~m}, \mathrm{CF}_{2} \mathrm{CF}_{2}\right), 171.6(\mathrm{~s}, 2 \times \mathrm{C}=\mathrm{O}), 184.2\left(\mathrm{t},{ }^{2} \mathrm{~J}_{\mathrm{C}, \mathrm{F}} 27.9 \mathrm{~Hz}, 2 \times \mathrm{C}=\mathrm{S}\right) . \mathrm{MS}, \mathrm{m} / \mathrm{z}$ : $393[\mathrm{M}+\mathrm{H}]^{+}, 391[\mathrm{M}-\mathrm{H}]^{-}$. Anal. calcd for $\mathrm{C}_{12} \mathrm{H}_{16} \mathrm{~F}_{4} \mathrm{~N}_{2} \mathrm{O}_{4} \mathrm{~S}_{2}: \mathrm{C}, 36.73 ; \mathrm{H}, 4.11 ; \mathrm{N}, 7.14 ; \mathrm{S}, 16.34$. Found: $\mathrm{C}, 36.76 ; \mathrm{H}$, 4.17; N, 7.18; S, 16.29.

Diisopropyl $N, N^{\prime}-(2,2,3,3-t e t r a f l u o r o b u t a n e d i t h i o y l) ~ b i s(L-v a l i n a t e)(7 c)$. Yield: $65 \%$. Yellow oil. $[\alpha]_{D}^{20}-119.0^{\circ}$ (c=1, MeOH). $R_{\mathrm{f}}=0.55$ (hexane/EtOAc 4:1). ${ }^{1} \mathrm{H} \mathrm{NMR}\left(\mathrm{CDCl}_{3}, \delta \mathrm{ppm}\right): 0.97\left(\mathrm{~d}, 3^{3} \mathrm{H}_{\mathrm{H}} \mathrm{H} 6.8 \mathrm{~Hz}, 6 \mathrm{H}, 2 \times \mathrm{CHCH}\left(\mathrm{CH}_{3}\right)_{2}\right)$, $1.07\left(\mathrm{~d}, 3^{3} \mathrm{~J}_{\mathrm{H}, \mathrm{H}} 6.8 \mathrm{~Hz}, 6 \mathrm{H}, 2 \times \mathrm{CHCH}\left(\mathrm{CH}_{3}\right)_{2}\right), 1.30\left(\mathrm{~m}, 12 \mathrm{H}, 2 \times \mathrm{OCH}\left(\mathrm{CH}_{3}\right)_{2}\right), 2.40\left(\mathrm{~m}, 2 \mathrm{H}, 2 \times \mathrm{CHC} \underline{\mathrm{H}}\left(\mathrm{CH}_{3}\right)_{2}\right), 4.91(\mathrm{~m}$, $\left.2 \mathrm{H}, 2 \times \underline{\mathrm{CHCH}}\left(\mathrm{CH}_{3}\right)_{2}\right), 5.12\left(\mathrm{~m}, 2 \mathrm{H}, 2 \times \mathrm{OC} \underline{\mathrm{H}}\left(\mathrm{CH}_{3}\right)_{2}\right), 8.47(\mathrm{bs}, 2 \mathrm{H}, 2 \times \mathrm{NH}) .{ }^{19} \mathrm{~F} \mathrm{NMR}\left(\mathrm{CDCl}_{3}, \delta \mathrm{ppm}\right):-108.8(\mathrm{dm}$, 
$\left.{ }^{2} J_{F, F} 248.1 \mathrm{~Hz}, 2 \mathrm{~F}, 2 \times \mathrm{CF}_{A} \mathrm{CF}_{B}\right),-111.5\left(\mathrm{dm},{ }^{2} \mathrm{~J}_{\mathrm{F}, \mathrm{F}} 248.1 \mathrm{~Hz}, 2 \mathrm{~F}, 2 \times \mathrm{CF}_{\mathrm{A}} \mathrm{CF}_{\mathrm{B}}\right) .{ }^{13} \mathrm{C} \mathrm{NMR}\left(\mathrm{CDCl}_{3}, \delta \mathrm{ppm}\right): 18.4(\mathrm{~s}$, $\left.\mathrm{CHCH}\left(\underline{\mathrm{CH}}_{3}\right)_{2}\right), 18.9\left(\mathrm{~s}, \mathrm{CHCH}\left(\underline{\mathrm{CH}}_{3}\right)_{2}\right), 21.9\left(\mathrm{~s}, 2 \times \mathrm{OCH}\left(\underline{\mathrm{CH}}_{3}\right)_{2}\right), 31.6\left(\mathrm{~s}, 2 \times \mathrm{CH} \underline{\mathrm{CH}}\left(\mathrm{CH}_{3}\right)_{2}\right), 62.9\left(\mathrm{~s}, 2 \times \underline{\mathrm{CHCH}}\left(\mathrm{CH}_{3}\right)_{2}\right)$, $70.2\left(\mathrm{~s}, 2 \times \mathrm{O} \underline{\mathrm{CH}}\left(\mathrm{CH}_{3}\right)_{2}\right), 109.1-114.6\left(\mathrm{~m}, \mathrm{CF}_{2} \mathrm{CF}_{2}\right), 169.2$ (s, $\left.2 \times \mathrm{C}=\mathrm{O}\right), 184.4\left(\mathrm{t},{ }^{2} \mathrm{~J}_{\mathrm{C}, \mathrm{F}} 26.7 \mathrm{~Hz}, 2 \times \mathrm{C}=\mathrm{S}\right) . \mathrm{MS}, \mathrm{m} / \mathrm{z}$ : $506[\mathrm{M}+\mathrm{H}]^{+}, 504[\mathrm{M}-\mathrm{H}]^{-}$. Anal. calcd for $\mathrm{C}_{20} \mathrm{H}_{32} \mathrm{~F}_{4} \mathrm{~N}_{2} \mathrm{O}_{4} \mathrm{~S}_{2}: \mathrm{C}, 47.60 ; \mathrm{H}, 6.39 ; \mathrm{N}, 5.55 ; \mathrm{S}, 12.71$. Found: $\mathrm{C}, 47.72 ; \mathrm{H}$, 6.45; N, 5.60; S, 12.86 .

2,2-Difluoromalonothioamide (6). Phosphorus pentasulfide (9.66 g, $21.7 \mathrm{mmol}$ ) and hexamethyldisiloxane (HMDSO) (5.64 g, $34.8 \mathrm{mmol}$ ) were added to a suspension of 2,2-difluoromalonoamide (5) (3.00 g, $21.7 \mathrm{mmol})$ in toluene $(50 \mathrm{~mL})$. The reaction mixture was stirred at $120{ }^{\circ} \mathrm{C}$ for $24 \mathrm{~h}$; the reaction was followed by ${ }^{19} \mathrm{~F} N M R$, monitoring the disappearance of the starting amide peak in the precipitate. The mixture was cooled to room temperature. The solid material was filtered off and washed with diethyl ether $(15 \mathrm{~mL})$. Solvents and excess HMDSO were removed in vacuo. The residue was purified by column chromatography on silica gel, eluting with a mixture of hexane and ethyl acetate (1:1). The resulting product was further recrystallized from chloroform giving 2,2-difluoromalonothioamide (6). Yield: $65 \%$. Yellow crystals, $\mathrm{mp} 135-136{ }^{\circ} \mathrm{C} . R_{\mathrm{f}}=0.74$

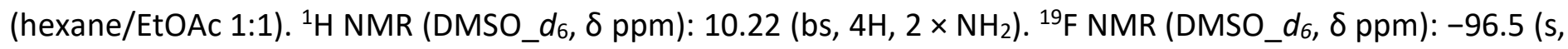
$\left.\mathrm{CF}_{2}\right) .{ }^{13} \mathrm{C}$ NMR (DMSO_d $\left.d_{6} \delta \mathrm{ppm}\right): 112.9\left(\mathrm{t},{ }^{1} \mathrm{~J}_{\mathrm{C}, \mathrm{F}} 258.5 \mathrm{~Hz}, \mathrm{CF}_{2}\right), 191.6\left(\mathrm{t},{ }^{2} \mathrm{~J}_{\mathrm{C}, \mathrm{F}} 27.9 \mathrm{~Hz}, 2 \times \mathrm{C}=\mathrm{S}\right) . \mathrm{MS}, \mathrm{m} / \mathrm{z}: 171$ $[\mathrm{M}+\mathrm{H}]^{+}$. Anal. calcd for $\mathrm{C}_{3} \mathrm{H}_{4} \mathrm{~F}_{2} \mathrm{~N}_{2} \mathrm{~S}_{2}: \mathrm{C}, 21.17 ; \mathrm{H}, 2.37 ; \mathrm{N}, 16.46 ; \mathrm{S}, 37.67$. Found: $\mathrm{C}, 21.19 ; \mathrm{H}, 2.41 ; \mathrm{N}, 16.50 ; \mathrm{S}$, 37.72.

General procedure for the reaction of 2,2-difluoromalonothioamide (6) with $\alpha$-amino acid esters. The respective amino acid ester $(2.7 \mathrm{mmol})$ was added to a solution of 2,2-difluoromalonothioamide (6) (200 mg, $1.2 \mathrm{mmol})$ in diethyl ether $(20 \mathrm{~mL})$. The reaction mixture was stirred at room temperature for 20 days. The solvent was then evaporated in vacuo and the crude product was purified by column chromatography on silica gel, eluting with a mixture of hexane and ethyl acetate (7:3) to afford the corresponding polyfluoroalkanedithioyl derivative (8a-c).

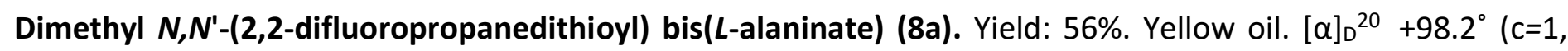
$\mathrm{MeOH}$ ). $R_{\mathrm{f}}=0.30$ (hexane/EtOAc 7:3). ${ }^{1} \mathrm{H} \mathrm{NMR}\left(\mathrm{CDCl}_{3}, \delta \mathrm{ppm}\right): 1.56\left(\mathrm{~d},{ }^{3} \mathrm{~J}_{\mathrm{H}, \mathrm{H}} 6.9 \mathrm{~Hz}, 6 \mathrm{H}, 2 \times \mathrm{CH}_{3}\right), 3.82(\mathrm{~s}, 6 \mathrm{H}, 2 \times$ $\left.\mathrm{OCH}_{3}\right), 5.00(\mathrm{~m}, 2 \mathrm{H}, 2 \times \mathrm{CH}), 8.78$ (bs, $\left.2 \mathrm{H}, 2 \times \mathrm{NH}\right) .{ }^{19} \mathrm{~F} \mathrm{NMR}\left(\mathrm{CDCl}_{3}, \delta \mathrm{ppm}\right):-97.0\left(\mathrm{~s}, \mathrm{CF}_{2}\right) .{ }^{13} \mathrm{C} \mathrm{NMR}\left(\mathrm{CDCl}_{3}, \delta\right.$ ppm): $16.2\left(\mathrm{~s}, 2 \times \mathrm{CH}_{3}\right), 53.1\left(\mathrm{~s}, 2 \times \mathrm{OCH}_{3}\right), 53.6(\mathrm{~s}, 2 \times \mathrm{CH}), 112.6\left(\mathrm{t},{ }^{1} \mathrm{~J}_{\mathrm{C}, \mathrm{F}} 257.8 \mathrm{~Hz}, \mathrm{CF}_{2}\right), 171.6(\mathrm{~s}, 2 \times \mathrm{C}=\mathrm{O}), 187.9$ $\left(t,{ }^{2} J_{C, F} 27.9 \mathrm{~Hz}, 2 \times \mathrm{C}=\mathrm{S}\right)$. MS, $\mathrm{m} / \mathrm{z}: 343[\mathrm{M}+\mathrm{H}]^{+}, 341[\mathrm{M}-\mathrm{H}]^{-}$. Anal. calcd for $\mathrm{C}_{11} \mathrm{H}_{16} \mathrm{~F}_{2} \mathrm{~N}_{2} \mathrm{O}_{4} \mathrm{~S}_{2}: \mathrm{C}, 38.59 ; \mathrm{H}, 4.71 ; \mathrm{N}$, 8.18; S, 18.73. Found: C, 38.60; H, 4.67; N, 8.21; S, 18.75 .

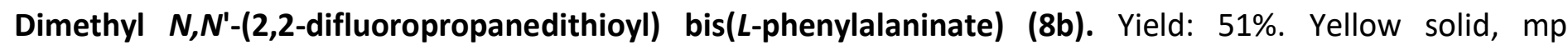
$108-110^{\circ} \mathrm{C} .[\alpha]_{\mathrm{D}}{ }^{20}-76.3^{\circ}$ (c=1, MeOH). $R_{\mathrm{f}}=0.63$ (hexane/EtOAc 7:3). ${ }^{1} \mathrm{H} \mathrm{NMR}\left(\mathrm{CDCl}_{3}, \delta \mathrm{ppm}\right): 3.24\left(\mathrm{dd},{ }^{2} J_{\mathrm{H}, \mathrm{H}}\right.$ $\left.14.0 \mathrm{~Hz},{ }^{3} J_{\mathrm{H}, \mathrm{H}} 4.8 \mathrm{~Hz}, 2 \mathrm{H}, 2 \times \underline{\mathrm{C}}_{\mathrm{A}} \mathrm{H}_{\mathrm{B}}\right), 3.35\left(\mathrm{dd},{ }^{2} \mathrm{~J}_{\mathrm{H}, \mathrm{H}} 14.0 \mathrm{~Hz},{ }^{3} J_{\mathrm{H}, \mathrm{H}} 4.8 \mathrm{~Hz}, 2 \mathrm{H}, 2 \times \mathrm{CH}_{\mathrm{A}} \underline{H}_{\mathrm{B}}\right), 3.74\left(\mathrm{~s}, 6 \mathrm{H}, 2 \times \mathrm{OCH}_{3}\right)$, $5.29(\mathrm{~m}, 2 \mathrm{H}, 2 \times \mathrm{CH}), 7.14-7.31(\mathrm{~m}, 10 \mathrm{H}, 2 \times \mathrm{Ph}), 8.67$ (bs, $2 \mathrm{H}, 2 \times \mathrm{NH}) .{ }^{19} \mathrm{~F} \mathrm{NMR}\left(\mathrm{CDCl}_{3}, \delta \mathrm{ppm}\right):-98.9\left(\mathrm{~s}, \mathrm{CF}_{2}\right)$. ${ }^{13} \mathrm{C} \mathrm{NMR}\left(\mathrm{CDCl}_{3}, \delta \mathrm{ppm}\right): 36.1\left(\mathrm{~s}, 2 \times \mathrm{CH}_{2}\right), 52.8(\mathrm{~s}, 2 \times \mathrm{CH}), 58.6\left(\mathrm{~s}, 2 \times \mathrm{OCH}_{3}\right), 112.4\left(\mathrm{t},{ }^{1} \mathrm{~J}_{\mathrm{C}, \mathrm{F}} 260.2 \mathrm{~Hz}, \mathrm{CF}_{2}\right), 127.6$ (s, $2 \times \mathrm{CH}$ Ph), 128.9 (s, $4 \times \mathrm{CH} P h), 129.6$ (s, $4 \times \mathrm{CH} \mathrm{Ph}), 134.7$ (s, $\left.2 \times \mathrm{C}_{\mathrm{q}} \mathrm{Ph}\right), 170.0(\mathrm{~s}, 2 \times \mathrm{C}=\mathrm{O}), 188.0\left(\mathrm{t},{ }^{2} \mathrm{~J}_{\mathrm{C}, \mathrm{F}}\right.$ $28.4 \mathrm{~Hz}, 2 \times \mathrm{C}=\mathrm{S})$. MS (EI): $\mathrm{m} / \mathrm{z} 496[\mathrm{M}+\mathrm{H}]^{+}, 494[\mathrm{M}-\mathrm{H}]^{-}$. Anal. calcd for $\mathrm{C}_{23} \mathrm{H}_{24} \mathrm{~F}_{2} \mathrm{~N}_{2} \mathrm{O}_{4} \mathrm{~S}_{2}: \mathrm{C}, 55.86 ; \mathrm{H}, 4.89 ; \mathrm{N}$, 5.66; S, 12.96 . Found: C, 55.90; H, 4.87; N, 5.64; S, 13.00.

Diisopropyl N,N'-(2,2-difluoropropanedithioyl) bis(L-valinate) (8c). Yield: $68 \%$. Yellow oil. [ $\alpha]_{\mathrm{D}}{ }^{20}-187.4^{\circ}(\mathrm{c}=1$, MeOH). $R_{\mathrm{f}}=0.56$ (hexane/EtOAc 4:1). ${ }^{1} \mathrm{H} \mathrm{NMR}\left(\mathrm{CDCl}_{3}, \delta \mathrm{ppm}\right): 0.99\left(\mathrm{~d}, 3^{3} \mathrm{H}_{\mathrm{H}} 6.8 \mathrm{~Hz}, 6 \mathrm{H}, \mathrm{CHCH}\left(\mathrm{C}_{3}\right)_{2}\right), 1.07(\mathrm{~d}$, $\left.3^{3} \mathrm{H}_{, \mathrm{H}} 6.8 \mathrm{~Hz}, 6 \mathrm{H}, \mathrm{CHCH}\left(\mathrm{CH}_{3}\right)_{2}\right), 1.30\left(\mathrm{~m}, 12 \mathrm{H}, 2 \times \mathrm{OCH}\left(\mathrm{CH}_{3}\right)_{2}\right), 2.42\left(\mathrm{~m}, 2 \mathrm{H}, 2 \times \mathrm{CHCH}\left(\mathrm{CH}_{3}\right)_{2}\right), 4.96(\mathrm{~m}, 2 \mathrm{H}, 2 \times$ $\left.\mathrm{CHCH}\left(\mathrm{CH}_{3}\right)_{2}\right), 5.13\left(\mathrm{~m}, 2 \mathrm{H}, 2 \times \mathrm{OC} \underline{\mathrm{H}}\left(\mathrm{CH}_{3}\right)_{2}\right), 8.72(\mathrm{bs}, 2 \mathrm{H}, 2 \times \mathrm{NH}) .{ }^{19} \mathrm{~F} \mathrm{NMR}\left(\mathrm{CDCl}_{3}, \delta \mathrm{ppm}\right):-100.6\left(\mathrm{~s}, \mathrm{CF}_{2}\right) .{ }^{13} \mathrm{C}$ $\mathrm{NMR}\left(\mathrm{CDCl}_{3}, \delta \mathrm{ppm}\right): 18.5\left(\mathrm{~s}, \mathrm{CHCH}\left(\underline{C H}_{3}\right)_{2}\right), 18.6\left(\mathrm{~s}, \mathrm{CHCH}\left(\underline{C H}_{3}\right)_{2}\right), 21.9\left(\mathrm{~s}, 2 \times \mathrm{OCH}\left(\underline{C H}_{3}\right)_{2}\right), 31.4(\mathrm{~s}, 2 \times$ $\left.\mathrm{CH} \underline{\mathrm{CH}}\left(\mathrm{CH}_{3}\right)_{2}\right), 62.7\left(\mathrm{~s}, 2 \times \underline{\mathrm{C}} \mathrm{HCH}\left(\mathrm{CH}_{3}\right)_{2}\right), 70.0\left(\mathrm{~s}, 2 \times \mathrm{O} \underline{\mathrm{CH}}\left(\mathrm{CH}_{3}\right)_{2}\right), 112.9\left(\mathrm{t},{ }^{1} \mathrm{~J}_{\mathrm{C}, \mathrm{F}} 258.8 \mathrm{~Hz}, \mathrm{CF}_{2}\right), 169.2(\mathrm{~s}, 2 \times \mathrm{C}=\mathrm{O})$, 
$188.6\left(\mathrm{t},{ }^{2} \mathrm{~J}_{\mathrm{C}, \mathrm{F}} 28.0 \mathrm{~Hz}, 2 \times \mathrm{C}=\mathrm{S}\right) . \mathrm{MS}, \mathrm{m} / \mathrm{z}: 456[\mathrm{M}+\mathrm{H}]^{+}, 454[\mathrm{M}-\mathrm{H}]^{-}$. Anal. calcd for $\mathrm{C}_{19} \mathrm{H}_{32} \mathrm{~F}_{2} \mathrm{~N}_{2} \mathrm{O}_{4} \mathrm{~S}_{2}: \mathrm{C}, 50.20 ; \mathrm{H}$, 7.10; N, 6.16; S, 14.10. Found: C, 50.28; H, 6.98; N, 6.18; S, 14.19.

General procedure for the reaction of 2,2-difluoromalonothioamide (6) with dipeptide esters. The respective dipeptide ester $(2.7 \mathrm{mmol})$ was added to a solution of 2,2-difluoromalonothioamide (6) (200 $\mathrm{mg}, 1.2 \mathrm{mmol})$ in toluene $(15 \mathrm{~mL})$. The reaction mixture was heated at $80^{\circ} \mathrm{C}$ for $50 \mathrm{~h}$. Then the solvent was evaporated in vacuo and the crude product was purified by column chromatography on silica gel, eluting with a mixture of hexane and ethyl acetate (1:1) to afford the corresponding polyfluoroalkanedithioyl derivative $(9 \mathbf{a}, \mathbf{b})$ as a mixture of diastereomers.

Dimethyl $N, N^{\prime}$-(2,2-difluoropropanedithioyl) bis( $D L-\alpha$-alanyl-DL-valinate) (9a). Yield: 58\%. Brown oil. $R_{\mathrm{f}}=0.63$ (hexane/EtOAc 1:1). Mixture of diastereomers (ratio of 50:50 according to ${ }^{19} \mathrm{~F} \mathrm{NMR).}{ }^{1} \mathrm{H} N M R\left(\mathrm{CDCl}_{3}, \delta \mathrm{ppm}\right): 0.89$ (d, $\left.{ }^{3} J_{\mathrm{H}, \mathrm{H}} 6.4 \mathrm{~Hz}, 6 \mathrm{H}, \mathrm{CH}\left(\mathrm{CH}_{3}\right)_{2}\right), 0.94\left(\mathrm{~d},{ }^{3} J_{\mathrm{H}, \mathrm{H}} 6.4 \mathrm{~Hz}, 6 \mathrm{H}, \mathrm{CH}\left(\mathrm{CH}_{3}\right)_{2}\right), 1.59\left(\mathrm{~m}, 6 \mathrm{H}, 2 \times \mathrm{CHCH}_{3}\right), 2.17\left(\mathrm{~m}, 2 \mathrm{H}, 2 \times \mathrm{C} \underline{\mathrm{H}}\left(\mathrm{CH}_{3}\right)_{2}\right), 3.76(\mathrm{~s}$, $\left.6 \mathrm{H}, 2 \times \mathrm{OCH}_{3}\right), 4.58\left(\mathrm{~m}, 2 \mathrm{H}, 2 \times \mathrm{CH}_{\mathrm{H}} \mathrm{COOMe}\right), 5.00\left(\mathrm{~m}, 2 \mathrm{H}, 2 \times \mathrm{CHCH}_{3}\right), 6.76\left(\mathrm{~d},{ }^{3} J_{\mathrm{H}, \mathrm{H}} 8.8 \mathrm{~Hz}, 2 \mathrm{H}, 2 \times \mathrm{C}(\mathrm{S}) \mathrm{NH}\right), 9.09(\mathrm{bs}, 2 \mathrm{H}, 2$ $\times \mathrm{C}(\mathrm{O}) \mathrm{NH}) .{ }^{19} \mathrm{~F} \mathrm{NMR}\left(\mathrm{CDCl}_{3}, \delta \mathrm{ppm}\right):-99.6\left(\mathrm{dm},{ }^{2} J_{\mathrm{F}, \mathrm{F}} 229.3 \mathrm{~Hz}, 1 \mathrm{~F}, \mathrm{CF}_{\mathrm{A}} \mathrm{F}_{\mathrm{B}}\right),-99.7\left(\mathrm{~m}, 2 \mathrm{~F}, \mathrm{CF}_{2}\right),-100.8\left(\mathrm{dm},{ }^{2} J_{\mathrm{F}, \mathrm{F}} 229.3 \mathrm{~Hz}, 1 \mathrm{~F}\right.$, $\left.\mathrm{CF}_{A} \underline{F}_{B}\right) .{ }^{13} \mathrm{CNMR}\left(\mathrm{CDCl}_{3}, \delta \mathrm{ppm}\right): 17.2\left(\mathrm{~s}, \mathrm{CHCH}_{3}\right), 17.3\left(\mathrm{~s}, \mathrm{CHCH}_{3}\right), 18.0\left(\mathrm{~s}, \mathrm{CH}\left(\underline{\mathrm{CH}}_{3}\right)_{2}\right), 18.1\left(\mathrm{~s}, \mathrm{CH}\left(\underline{\mathrm{CH}}_{3}\right)_{2}\right), 19.1\left(\mathrm{~s}, \mathrm{CH}\left(\underline{\mathrm{CH}} \mathrm{H}_{3}\right)_{2}\right)$, $19.2\left(\mathrm{~s}, \underline{\mathrm{CH}}\left(\underline{\mathrm{CH}}_{3}\right)_{2}\right), 31.5\left(\mathrm{~s}, \underline{\mathrm{C}} \mathrm{H}\left(\mathrm{CH}_{3}\right)_{2}\right), 52.49\left(\mathrm{~s}, \underline{\mathrm{C}} \mathrm{HCH}_{3}\right), 52.53\left(\mathrm{~s}, \underline{\mathrm{C}} \mathrm{HCH}_{3}\right), 54.48\left(\mathrm{~s}, \underline{\mathrm{C}} \mathrm{HCH}_{3}\right), 54.53\left(\mathrm{~s}, \underline{\mathrm{C}} \mathrm{HCH}_{3}\right), 57.5(\mathrm{~s}$, $\left.\mathrm{OCH}_{3}\right), 113.1\left(\mathrm{t},{ }^{1} J_{\mathrm{C}, \mathrm{F}} 267.7 \mathrm{~Hz}, \mathrm{CF}_{2}\right), 170.1(\mathrm{~s}, \mathrm{C}=\mathrm{O}), 172.4(\mathrm{~s}, \mathrm{C}=\mathrm{O}), 172.4(\mathrm{~s}, \mathrm{C}=\mathrm{O}), 188.1\left(\mathrm{t},{ }^{2} J_{\mathrm{C}, \mathrm{F}} 26.6 \mathrm{~Hz}, \mathrm{C}=\mathrm{S}\right), 188.2\left(\mathrm{t},{ }^{2} J_{\mathrm{C}, \mathrm{F}}\right.$ $26.6 \mathrm{~Hz}, \mathrm{C}=\mathrm{S})$. MS, $\mathrm{m} / \mathrm{z}: 541[\mathrm{M}+\mathrm{H}]^{+}$, $539[\mathrm{M}-\mathrm{H}]^{-}$. Anal. calcd for $\mathrm{C}_{21} \mathrm{H}_{34} \mathrm{~F}_{2} \mathrm{~N}_{4} \mathrm{O}_{6} \mathrm{~S}_{2}: \mathrm{C}, 46.65 ; \mathrm{H}, 6.34 ; \mathrm{N}, 10.36 ; \mathrm{S}, 11.86$. Found: C, 46.72; H, 6.40; N, 10.41; S, 11.92 .

Dimethyl $N, N^{\prime}-(2,2-d i f l u o r o p r o p a n e d i t h i o y l) ~ b i s\left(D L-\alpha\right.$-alanyl- $\beta$-alaninate) (9b). Yield: $47 \%$. Brown oil. $R_{f}=$ 0.43 (hexane/EtOAc 1:1). Mixture of diastereomers (the ratio is 50:50 according to $\left.{ }^{19} \mathrm{~F} \mathrm{NMR}\right) .{ }^{1} \mathrm{H} \mathrm{NMR}\left(\mathrm{CDCl}_{3}, \delta\right.$ ppm): $1.54\left(\mathrm{~m}, 6 \mathrm{H}, 2 \times \mathrm{CH}_{3}\right), 2.56\left(\mathrm{~m}, 4 \mathrm{H}, 2 \times \mathrm{CH}_{2} \mathrm{C}(\mathrm{O})\right), 3.54\left(\mathrm{~m}, 4 \mathrm{H}, 2 \times \mathrm{NCH}_{2}\right), 3.70\left(\mathrm{~s}, 6 \mathrm{H}, 2 \times \mathrm{OCH}_{3}\right), 4.83(\mathrm{~m}$, $2 \mathrm{H}, 2 \times \mathrm{CH}), 6.57(\mathrm{~m}, 2 \mathrm{H}, 2 \times \mathrm{C}(\mathrm{S}) \mathrm{NH}), 8.89(\mathrm{~m}, 2 \mathrm{H}, 2 \times \mathrm{C}(\mathrm{O}) \mathrm{NH}) .{ }^{19} \mathrm{~F} \mathrm{NMR}\left(\mathrm{CDCl}_{3}, \delta \mathrm{ppm}\right):-99.9\left(\mathrm{~m}, 2 \mathrm{~F}, \mathrm{CF}_{2}\right)$, $-101.1\left(\mathrm{dm},{ }^{2} J_{F, F} 229.6 \mathrm{~Hz}, 1 \mathrm{~F}, \underline{C F}_{A} F_{B}\right),-100.2\left(\mathrm{dm},{ }^{2} J_{F, F} 229.6 \mathrm{~Hz}, 1 \mathrm{~F}, \mathrm{CF}_{A} \underline{F}_{B}\right) . M S, m / z: 483$ [M-H]-. Anal. Calcd for $\mathrm{C}_{17} \mathrm{H}_{26} \mathrm{~F}_{2} \mathrm{~N}_{4} \mathrm{O}_{6} \mathrm{~S}_{2}$ : C, 42.14; $\mathrm{H}, 5.41 ; \mathrm{N}, 11.56 ; \mathrm{S}, 13.24$. Found: $\mathrm{C}, 42.22 ; \mathrm{H}, 5.48 ; \mathrm{N}, 11.60 ; \mathrm{S}, 13.2$

\section{Supplementary Material}

${ }^{19} \mathrm{~F} N M R,{ }^{1} \mathrm{H} \quad \mathrm{NMR}$, and ${ }^{13} \mathrm{C}$ NMR Spectra of dimethyl $\mathrm{N}, \mathrm{N}$-(2,2-difluoropropanedithioyl) bis(Lphenylalaninate) (8b) (in $\left.\mathrm{CDCl}_{3}\right) ;{ }^{19} \mathrm{~F} \mathrm{NMR},{ }^{1} \mathrm{H} \mathrm{NMR}$, and ${ }^{13} \mathrm{C} \mathrm{NMR}$ Spectra of dimethyl $\mathrm{N}, \mathrm{N}^{1}-(2,2-$ difluoropropanedithioyl) bis(DL- $\alpha$-alanyl- $\beta$-alaninate) (9a) (in $\mathrm{CDCl}_{3}$ ).

\section{References}

1. Asante, V.; Mortier, J.; Wolber G.; Koksch, B. Amino Acids 2014, 46, 2733. http://dx.doi.org/10.1007/s00726-014-1819-7

2. Fustero, S. ; Sanchez-Rosello, M.; Baez,C.; Pozo, C.; Ruano, J.L.G.; Aleman, J.; Marzo I. ; Parra, A. Amino Acids 2011, 41, 559. http://dx.doi.org/10.1007/s00726-011-0881-7

3. O’Connor, N.K.; Hudson,A.S; Cobb,S.L.; O’Neil, D.; Robertson, J.; Duncan, V.; Murphy, C.D. Amino Acids 2014, $46,2745$. http://dx.doi.org/10.1007/s00726-014-1830-z

4. Devillers, E.; Pytkowicz, J.; Chelain, E.; Brigaud, T. Amino Acids 2016, 48, 1475. 
https://doi.org/10.1007/s00726-016-2200-9

5. Pfund, E. ; Lequeux, T.; Masson, S. ; Vazeux, M. ; Cordi, A.; Pierre, A. ; Serre, V.; Herve, G. Biorg. Med. Chem. 2005, 13, 4921.

http://dx.doi.org/10.1016/i.bmc.2005.05.026

6. Andersen, T.P.; Ghattas,A.G.; Lawesson, S-O. Tetrahedron 1983, 39, 3419.

https://doi.org/10.1016/S0040-4020(01)91595-9

7. Bagley, M.C., Dale, J.W., Merritt, E.A., Xiong, X. Chem. Rev. 2005,105, 685-714.

https://doi.org/10.1021/cr0300441

8. Nicolaou, K.C.; Zak, M. ; Rahimipour, S. ; Estrada, A.A. ; Lee, S.H. ; O’Brate, A. ; Giannakakou, P. ; Ghadiri, M.R. J. Am. Chem. Soc. 2005,127,15042-15044.

https://doi.org/10.1021/ja0552803

9. Radhakrishnan, S.K. ; Bhat, U.G. ; Hughes, D.E. ; Wang, I.-C. ; Costa, R.H. ; Gartel, A.L. Cancer Res. 2006, 66, 9731-9735.

https://doi.org/10.1158/0008-5472.CAN-06-1576

10. Kwok, J.M.-M. ; Myatt, S.S. ; Marson, C.M. ; Coombes, R.C. ; Constantinidou, D. ; Lam, E.W.-F. Mol. Cancer Ther. 2008, 7, 2022-2032.

https://doi.org/10.1158/1535-7163.MCT-08-0188

11. Bhat, U.G. ; Zipfel, P.A. ; Tyler, D.S. ; Gartel, A.L. Cell Cycle. 2008, 7, 1851-1855.

https://doi.org/10.4161/cc.7.12.6032

12. Hegde, N.S. ; Sanders, D.A. ; Rodriguez, R. ; Balasubramanian, S. Nat. Chem. 2011, 3, 725-731. https://doi.org/10.1038/nchem.1114

13. Sullivan, M. ; Li J. ; Kumar, S. ; Rogers, M.J. ; McCutchan, T.F. Mol. Biochem. Parasitol. 2000, 109, $17-23$. https://doi.org/10.1016/S0166-6851(00)00226-7

14. Schlitzer, M. ChemMedChem. 2007, 2, 944-986.

https://doi.org/10.1002/cmdc.200600240

15. Schoof, S. ; Pradel, G. ; Aminake, M.N. ; Ellinger, B. ; Baumann, S. ; Potowski, M. ; Najajreh, Y. ; Kirschner, M. ; Arndt, H.-D. Angew. Chem. Int. Ed. 2010, 49, 3317-3321.

https://doi.org/10.1002/anie.200906988

16. Motoi, U. ; Furukawa, S. ; Abe, F. ; Ushioda, M. ; Fujine, K. ; Johki, S. ; Hatori, H. ; Ueda, J. J. Antibiot. 2004, 57, 590-596.

https://doi.org/10.7164/antibiotics.57.590

17. Aoki, M. ; Ohtsuka, T. ; Yamada, M. ; Ohba, Y. ; Yoshizaki, H. ; Yasuno, H. ; Sano, T. ; Watanabe, J. ; Yokose, K. ; Roche, N. J. Antibiot. 1991, 44, 582-588.

https://doi.org/10.7164/antibiotics.44.582

18. Hashimoto, M. ; Murakami, T. ; Funahashi, K. ; Tokunaga, T. ; Nihei, K. ; Okuno, T. ; Kimura, T. ; Naoki, H. ; Himeno, H. Bioorg. Med. Chem. 2006, 14, 8259-8270.

https://doi.org/10.1016/j.bmc.2006.09.006

19. Mizuhara, N. ; Kuroda, M. ; Ogita, A. ; Tanaka, T. ; Usuki, Y. ; Fujita, K.-I. Bioorg. Med. Chem. 2011, 19, 5300-5310.

https://doi.org/10.1016/i.bmc.2011.08.010

20. Biffinger, J.C.; Kim H.W.; DiMango S.G. ChemBioChem 2004, 5, 622.

https://doi.org/10.1002/cbic.200300910

21. Yamazaki, T.; Taguchi, T.; Ojima, I. In Fluorine in medicinal chemistry and chemical biology (Ed.: I. Ojima), Blackwell Publishing, 2009, pp. 3-46. 
22. Uneyama, K. Eds. Organofluorine Chemistry, Blackwell: Oxford, 2006. https://doi.org/10.1002/9780470988589

23. Banks, R.E.; Smart, B.E.; Tatlow, J.C. Eds. Organofluorine chemistry: principles and commercial applications, Plenum: New York, 2000.

24. Filler, R.; Kobayashi, Y.; Yagupolskii, L.M. Eds. Organofluorine compounds in medicinal chemistry and biomedical applications, Elsevier: Amsterdam, 1993.

25. Hoeg-Jensen, T. Phosphorus, Sulfur and Silicon 1996, 108, 257. https://doi.org/10.1080/10426509608029658

26. Yang,E. ; Reese, M.R.; Humphrey, J.M. Org. Lett. 2012, 14, 3944. http://dx.doi.org/10.1016/i.bmc.2005.05.026

27. Garrett, G.S. ; Emge, T.J. ; Lee, S.C. ; Fischer, E.M. ;. Dyehouse, K. ; Mclver, J.M. J. Org. Chem. 1991, 56, 4823.

https://doi.org/10.1021/jo00016a004

28. Pikun, N.V.; Mykhaylychenko, S.S.; Kulik, I.B.; Shermolovich, Yu.G. J. Fluorine Chem. 2016, 185, 86. http://dx.doi.org/10.1016/j.jfluchem.2016.03.005 